\title{
Highly Potent Extracts from Pea (Pisum sativum) and Maize (Zea mays) Roots Can Be Used to Induce Quiescence in Entomopathogenic Nematodes
}

\author{
Geoffrey Jaffuel $^{1}$ • Ivan Hiltpold ${ }^{2} \cdot$ Ted C. J. Turlings $^{1}$
}

Received: 20 June 2015 /Revised: 25 August 2015 / Accepted: 27 August 2015 / Published online: 12 September 2015

(C) Springer Science+Business Media New York 2015

\begin{abstract}
Root exudates can play an important role in plantnematode interactions. Recent studies have shown that the root cap exudates obtained from several plant species trigger a state of dormancy or quiescence in various genera of nematodes. This phenomenon is not only of fundamental ecological interest, but also has application potential if the plant-produced compound(s) could be used to control harmful nematodes or help to prolong the shelf-life of beneficial entomopathogenic nematodes (EPNs). The identification of the compound(s) involved in quiescence induction has proven to be a major challenge and requires large amounts of active material. Here, we present a high-throughput method to obtain bioactive root extracts from flash-frozen root caps of green pea and maize. The root cap extract obtained via this method was considerably more potent in inducing quiescence than exudate obtained by a previously used method, and consistently induced quiescence in the EPN Heterorhabditis megidis, even after a 30-fold dilution. Extracts obtained from the rest of the root were equally effective in inducing quiescence. Infective juveniles (IJs) of $H$. megidis exposed to these extracts readily recovered from their quiescent state as soon as they were placed in moist soil, and they were at least as infectious as the IJs that had been stored in water. Excessive exposure of IJs to air interfered with the triggering of quiescence. The implications of these results and the next steps towards identification of the quiescence-inducing compound(s) are discussed from the perspective of applying EPN against soil-dwelling insect pests.
\end{abstract}

Ted C. J. Turlings

ted.turlings@unine.ch

1 FARCE Laboratory, Institute of Biology, University of Neuchâtel, Rue Emile-Argand 11, 2000 Neuchâtel, Switzerland

2 Hawkesbury Institute for the Environment, University of Western Sydney, Locked Bag 1797, Penrith, NSW 2751, Australia
Keywords Entomopathogenic nematodes $\cdot$ Quiescence factor $\cdot$ Exudate $\cdot$ Extract $\cdot$ EPN formulation $\cdot$ Crop pests

\section{Introduction}

Plants produce a wide range of chemical organic compounds. When released into the environment, these metabolites mediate interactions with surrounding organisms (Barber et al. 1976), and can play major roles in defense, communication, attraction, and repellency (Vining 1990). Aboveground interactions that are mediated by plant compounds generally are well documented, but it is only recently that the role of rootproduced compounds in belowground interactions is receiving equal attention (Hartmann 2007; Hiltpold et al. 2011; Rasmann et al. 2012; Turlings et al. 2012; van Dam 2009). This seems pertinent because at least $20 \%$ of the photosynthetically assimilated carbon is released by the roots (Barber et al. 1976; Kumar et al. 2006). Several effects of root-released chemicals on nematodes, bacteria, and fungi have been described (Bais et al. 2006), and evidence is accumulating that they also serve an important function in belowground tritrophic interactions among plants, herbivores, and entomopathogenic nematodes (Ali et al. 2010, 2012; Hiltpold and Turlings 2012; Rasmann et al. 2005).

Initial research on soil-dwelling nematodes and root exudates focused on plant-parasitic nematodes, due to their importance as pests of crops. Plant parasitic nematodes use constitutively released root exudates to locate their host plant (Curtis et al. 2009; Prot 1980; Reynolds et al. 2011; Rolfe et al. 2000). Root exudates also are known to trigger egg hatching in several plant-parasitic nematodes (Den Nijs and Lock 1992; Gaur et al. 2000; Khokon et al. 2009). Zhao et al. (2000) were the first to observe the intriguing phenomenon that root cap exudates induce a state of dormancy (quiescence) 
in a plant-parasitic nematode (Meloidogyne incognita), and they proposed it to be a defense mechanism against root tip penetration. Following up, Hubbard et al. (2005) found that the root cap exudates of a wide variety of plants can trigger quiescence in several species of plant parasitic nematodes, animal parasitic nematodes, and free living nematodes. At the time, only one species of entomopathogenic nematode (EPN), Steinernema glaseri, was tested and found to be susceptible to the exudates.

It also is known that roots under attack by herbivores release compounds that attract EPN as a secondary defense mechanism. For instance, when maize roots are damaged by the western corn rootworm, Diabrotica virgifera virgifera, they release $(E)$ - $\beta$-caryophyllene, a volatile sesquiterpene that attracts the EPN Heterorhabditis megidis (Rasmann et al. 2005). This apparent defense mechanism has been confirmed for other plants and EPN systems (Ali et al. 2010, 2012; Van Tol et al. 2001). More recently, several species of EPN also have been shown to be susceptible to root cap exudates. The exudates were found to induce quiescence in all tested EPN species, and their activity could be restored by diluting the exudate with water (Hiltpold et al. 2014).

Control of soil-dwelling insect pests mainly relies on pesticides causing environmental concerns (Köhler and Triebskorn 2013). Hence, there is a clear need for sustainable alternatives that are based on ecologically sound crop management solutions. Biological control with EPNs could offer a sustainable alternative to chemical pesticides, and thus have been under intensive research in this context (Lewis et al. 2006). The genera Steinernema and Heterorhabditis are particularly promising, as these obligate parasites of insects rapidly kill their host after initial infection (Lewis et al. 2006). The infective juveniles (IJs), the free-living stage of EPNs, have evolved various strategies to locate and enter an insect host. Once inside, they release their symbiotic bacteria in the homeocel, which produce toxins and cause a lethal septicemia within 2 to 3 days (Adams and Nguyen 2002; Dillman et al. 2012). The EPNs feed on the bacteria and reproduce into the cadaver. When the resource is depleted, a new generation of IJs is produced, and they leave the carcass and find new hosts (Dillman et al. 2012; Kaya and Gaugler 1993).

Despite their efficiency in killing insect hosts, the use of EPNs as biocontrol control agents for soil insect pests remains challenging. One of the primary constraints is their short shelf life. Infective juveniles survive about a month in refrigerated vermiculite formulations, which is the prevailing storage method of commercially available EPN (Shapiro-Ilan et al. 2006). Prolonged storage diminishes EPN quality and their ability to reach a good level of control of the target pest (Grewal 2002). Thus, storage limitation has been a critical aspect in EPN formulations. In addition, EPNs usually are sprayed on top of the soil, exposing them to UV light and desiccation (Lello et al. 1996). These constraints make the use of EPNs costly and only marginally effective in large scale application (Georgis et al. 2006). We propose that triggering a state of quiescence, to prolong shelf life, in combination with novel application methods may render EPNs much more effective as biological control agents.

The state of quiescence is characterized by a straight shape and non-motile state of nematodes. Quiescence normally is triggered by unfavorable environmental conditions, such as extreme temperature, lack of oxygen, a lack of moisture, and/or an osmotic stress (Barrett 1991). During this state of dormancy, the metabolism of nematodes is strongly reduced, allowing them to conserve energy, which can significantly prolong their lifespan and infectiousness (Hiltpold et al. 2014). Quiescence is reversible when the conditions turn more favorable. Quiescence also can be chemically triggered, for example with glycerol (Chen and Glazer 2005) or with compound(s) in exudates of root caps (Hiltpold et al. 2014; Hubbard et al. 2005; Zhao et al. 2000). The advantages of glycerol and the so-called quiescence factors $(\mathrm{QFs})$ are that they provide full control of the nematodes quiescence by dose regulation, as well as of the recovery by simple dilution. Hiltpold et al. (2014) showed that exposure to pea root cap exudates conserves EPN motility, infectiousness, and lipid content, which implies that including QFs in EPN formulations has potential to enhance their efficacy. It therefore is worthwhile to identify the QF. However, collecting exudate is a tedious and highly time-consuming process. Moreover, the QF concentration contained in the exudate is low and its activity is quickly lost after a few dilutions (Hiltpold et al. 2014). It is for this reason that we set out to develop a high-throughput collection method that allowed us to obtain root extracts from root cap of green pea and maize, which were flash-frozen in liquid nitrogen.

To demonstrate that the root cap extract contains high concentrations of the QF, we incubated the EPN Heterorhabditis megidis in different dilutions of root cap extract and measured quiescence levels. To further improve the collection of QF, we also tested the possibility of using an extract of the entire root germinate instead of only the root cap. We compared quiescence of $H$. megidis IJs induced by the two different extracts. Furthermore, we determined whether quiescent IJs can recover from their inactive state and still efficiently penetrate and kill a target host. Two hosts were tested, larvae of the highly susceptible wax moth, Galleria mellonella, and larvae of the more resistant mealworm, Tenebrio molitor (Grewal and Peters 2005). In these tests, we compared the infectiousness of $H$. megidis IJs that had recovered from quiescence after storage in different root cap extract concentrations for $24 \mathrm{hr}$ with the infectiousness of IJs that had been stored in water. Finally, because we hypothesized that quiescence is a response to oxygen deprivation, we also evaluated the effect of aeration of the storage solutions on the induction of quiescence in H. megidis. 


\section{Methods and Materials}

Plants, Nematodes and Insects Resources Pea (Pisum sativum L.) seeds (variety Lancet, Wyss Samen und Planzen AG, Switzerland) were first sterilized in $95 \%$ ethanol for $5 \mathrm{~min}$, then rinsed and immerged in distilled water for $12 \mathrm{hr}$. Soaked seeds were placed in plastic boxes $\left(15 \times 13.5 \times 5 \mathrm{~cm}^{3}\right)$ containing phytoagar $1.0 \%$ (Duchefa Biochemie, Haarlem, The Netherlands) and incubated at $25{ }^{\circ} \mathrm{C}$ in the dark for 3 days.

Maize (Zea mayz L.) seeds (cultivar Delprim, DSP SA, Switzerland) were sterilized in distilled water with $10 \%$ bleach for $12 \mathrm{hr}$. Soaked seeds were placed in plastic boxes $\left(30 \times 30 \times 10 \mathrm{~cm}^{3}\right) \mathrm{kept}$ moist on paper towels, and incubated at $25^{\circ} \mathrm{C}$ in the dark for 3 days.

Heterorhabditis megidis was obtained from Andermatt Biocontrol SA, Switzerland. The EPNs were reared in the laboratory by infecting Galleria mellonella L. (Lepidoptera: Pyralidae) larvae, and new generations of IJs were recovered in White traps (White 1927) and stored at $10{ }^{\circ} \mathrm{C}$ before use. All experiments were performed with new, fresh IJs that were not more than 2-week-old.

One to 2-week-old larvae of the wax moth Galleria mellonella were used to rear the EPNs and for the infectiousness bioassays. These insects were obtained from Au Pêcheur SARL Neuchâtel (Switzerland). For the infectiousness test, we also used mealworm larvae (Tenebrio molitor), which were obtained from the University of Lausanne (Switzerland).

Extraction of Root Caps We collected $19.8 \mathrm{~g}$ (for maize) and $18.6 \mathrm{~g}$ (for pea) of the terminal $10 \mathrm{~mm}$ of the roots. This material was ground under liquid nitrogen in a pre-cooled mortar. The obtained extract was split equally into two $50 \mathrm{ml}$ Falcon tubes with $45 \mathrm{ml}$ of MilliQ water, and subjected to ultrasonication for $1.5 \mathrm{hr}$. The root cap extract then was centrifuged at $4500 \mathrm{rpm}$ for 10 min (Universal 16, Hettich Zentrifugen). The supernatants were pooled per plant species and filtered over a filter paper (Whatman $90 \mathrm{~mm} \varnothing, 4-7 \mu \mathrm{m}$ particle retention) in vacuo and lyophilized, which resulted in $1.49 \mathrm{~g}$ (maize) and $1.52 \mathrm{~g}$ (pea) of dry root cap extract. The dry extracts were stored at $-80^{\circ} \mathrm{C}$ until further use. For maize, we repeated the entire procedure with the remaining parts of the root (root extract), thus excluding the ten first millimeter of the root cap.

Collection of Root Cap Exudate Following Hubbard et al. (2005) and Hiltpold et al. (2014), the root cap exudate was collected from 15 pea germinates by placing the terminal $10 \mathrm{~mm}$ of the root caps in a $1 \mathrm{ml}$ drop of MilliQ water on a Teflon plate for $2 \mathrm{~min}$. Then, the water drop was collected and centrifuged at $14,000 \mathrm{rpm}$ for $10 \mathrm{~min}$ at $18^{\circ} \mathrm{C}$ (eppendorf $\mathrm{AG}$, centrifuge 5424), and the supernatant was stored at $-20{ }^{\circ} \mathrm{C}$ (root cap exudate).
Quiescence Induction Tests with $\boldsymbol{H}$. megidis The sensitivity of $H$. megidis to the QF in the root cap extract and the root cap exudate at different concentrations was assessed following a previously described method (Hubbard et al. 2005; Hiltpold et al. 2014). A suspension containing 30 IJs per $50 \mu$ of distilled water was prepared. Additionally, pea root cap extract was adjusted to seven different dilutions in MilliQ water: $10 \mathrm{mg}$ (not diluted), $2 \mathrm{mg}(\times 5), 1 \mathrm{mg}(\times 10), 0.66 \mathrm{mg}(\times 15)$, $0.5 \mathrm{mg}(\times 20), 0.4 \mathrm{mg}(\times 25), 0.33 \mathrm{mg}(\times 30)$. Root cap exudate already in solution was adjusted to obtain the same seven dilutions (not diluted, $\times 5, \times 10, \times 15, \times 20, \times 25$, and $\times 30$ ). The control contained only MilliQ water. Suspensions $(50 \mu \mathrm{l})$ with H. megidis IJs were poured into each well of a 96-well tissue plate (Sigma-Aldrich). Then, $175 \mu$ lof the different solutions of root cap extract or exudate were added, resulting in a total volume of $225 \mu \mathrm{l}$ per well. After $12 \mathrm{hr}$, we counted the number of quiescent IJs in the wells ( $N=10$ /dilutions) under a microscope. Immobile and straight-shaped IJs were considered to be quiescent. The experiment was repeated three times with different batches of IJs. The same bioassay was used to assess the induction of quiescence by maize germinate root caps $(0$ $10 \mathrm{~mm}, N=12)$ compared to the rest of the maize germinate $\operatorname{root}(>10 \mathrm{~mm}, N=12)$. For the maize extract, we tested a reduced number of dilutions (not diluted, $\times 5, \times 10, \times 20$, and $\times 30$ ). The experiment was replicated twice.

The percentages of quiescent EPN were compared using Wilcoxon Signed-Rank test performed in R version 2.15.2 (http://www.r-project.org/). Bonferroni correction was applied on the $P$-values to overcome multiple comparisons.

Recovery and Infectiousness of $\boldsymbol{H}$. megidis The ability of $H$. megidis to infect and kill a host depending on storage (for $24 \mathrm{hr}$ ) in different concentrations of root cap extract was compared to H. megidis stored in water only. We tested the infectiousness using highly susceptible $G$. mellonella larvae, as well as more resistant T. molitor larvae. Suspensions $(1.5 \mathrm{ml})$ of water with $H$. megidis at a concentration of 50 IJs in $10 \mu \mathrm{l}$ were centrifuged in a $1.5 \mathrm{ml}$ Eppendorf tube at $8000 \mathrm{rpm}$ for $5 \mathrm{~min}$ (Eppendorf AG, centrifuge 5424), and the supernatant was replaced by different concentrations of root extracts (pea and maize). The final solutions were transferred into $4 \mathrm{ml}$ glass tubes (BGB analytik, AG) for $24 \mathrm{hr}$ to trigger quiescence before inoculation. The different concentrations of extract were: $10 \mathrm{mg}$ (not diluted), $1 \mathrm{mg}(\times 10), 0.5 \mathrm{mg}(\times 20), 0.33 \mathrm{mg}$ $(\times 30)$, and water as control $(N=15 /$ concentration). Following Hiltpold et al. (2012), $50 \mathrm{ml}$ Falcon tubes were filled with $60 \%$ moist potting soil. One G. mellonella or one T. molitor larva was placed in an individual plastic specimen tube $(1.5 \mathrm{ml}$ Eppendorf tube pierced with 12 holes). The tubes containing the larvae then were each placed inside a $50 \mathrm{ml}$ Falcon tube, $3 \mathrm{~cm}$ below the soil surface. They were placed at the edge of the tube so that the larvae could be observed. To each Falcon tube we added $50 \mu \mathrm{l}$ of one of the dilutions, or water as control, 
containing IJs (ca. 250). Each solution was poured onto the soil, and a $1 \mathrm{~cm}$ soil layer was added to cover the drop. The tubes were not fully closed to allow gas exchange, and they were stored in the dark at room temperature. Every treatment was replicated 15 times. Every day, the success of $H$. megidis in infecting the larvae was evaluated for each tube by visual inspection, looking for red larvae, which is indicative of infection (Forst and Clarke 2002). Immobile larvae also were checked for mortality. Overall larval survival was evaluated 7 days after the start of an experiment. Parametric survival analyses were performed to evaluate significant differences in larval survival using the survival package of R program version 2.15.2 (http:// www.r-project.org/). The Weibull distribution of error produced the minimum error deviance and was therefore selected for further analyses.

Quiescence Factor Aeration To verify if an excess availability of oxygen interferes with the triggering of quiescence, a set of three experiments was performed. The general setup involved ca. $1000 \mathrm{IJ}$ 's in $50 \mathrm{ml}$ of water that were poured into a $4 \mathrm{ml}$ glass tube (BGB analytik, AG). After application of the treatments described below, tubes were covered with one layer of parafilm to avoid evaporation, but allowing gas exchange. The tubes were stored at room temperature, and after $24 \mathrm{hr}$ of exposure, quiescence was assessed.

In the first experiment, two different volumes of a solution of $1 \mathrm{mg}$ of maize root cap extract were added to the tubes, a large volume of $1 \mathrm{ml}(N=20)$ or a smaller volume of $200 \mu \mathrm{l}$ $(N=20)$.

In a second experiment, $1 \mathrm{ml}$ of a solution of maize root cap extract at a concentration of $1 \mathrm{mg}$ was added, half of the tubes were placed on an agitator to oxygenate the solution at $400 \mathrm{tr} /$ min (Edmund Bühler Compact Mixer Shaker KL-2) for $24 \mathrm{hr}$ $(N=20)$ and the other tubes $(N=20)$ were just placed on a shelf next to the agitator. All tubes were kept at room temperature.

In the third experiment, maize root cap extract was prepared in a $250 \mathrm{ml}$ Falcon tube and oxygenized for $3 \mathrm{hr}$ with an aquarium air pump, before IJs were placed in the solution.
In parallel, a similar solution was made without pump aeration. One $\mathrm{ml}$ of the oxygenized solution $(N=20)$ and nonoxygenized solution $(N=20)$ were added to the tubes with the IJs. Twenty vials containing only $1 \mathrm{ml}$ of water served as controls. The purpose of this latter experiment was to specifically test if the QF would become inactive after exposure to excess oxygen.

Every experiment was replicated twice. Binomial GLM performed in R version 2.15 .2 (http://www.r-project.org/) was used to assess difference between treatments.

\section{Results}

Extract Versus Exudate Root cap exudate was efficient only in inducing quiescence in $H$. megidis when not diluted, resulting in $90.34 \%$ of quiescent EPNs, whereas there was no quiescence in the water controls (Bonferroni corrected $P$-value $<0.001, W=900, P<0.001)$. All other root cap exudate dilutions were not significantly different from the water control $(\alpha<0.001 ; \times 5: W=309.5, P=0.03 ; \times 10: W=240.5$, $P=0.001 ; \times 15: W=337.5, P=0.09 ; \times 20: W=374, P=0.2$; $\times 25: W=278.5, P=0.01 ; \times 30: W=408.5, P=0.54)$. In contrast, root cap extract induced $100 \%$ quiescence even at a $15 \times$ dilution, and quiescence induction was not significantly different from the undiluted root cap extract up to the $25 \times$ dilution $(\alpha<0.001 ; \times 1, \times 5, \times 10$ and $\times 15$ induced $100 \%$ quiescence, $\times 20: W=525, P=0.02 ; \times 25: W=585, P=0.001 ; \times 30$ : $W=780, P<0.001)$. The undiluted root cap exudate was less effective in inducing quiescence than the undiluted root cap extract $(\alpha<0.001 ; W=765, P<0.001)$, this difference persisted until the $\times 20$ dilution of the extract $(\alpha<0.001 ; 25 \times$ : $W=609, P=0.012$; Fig. 1).

Maize Root Cap Extract Versus the Rest of Root Extract Extract obtained from maize roots also were efficient in inducing quiescence in H. megidis. Root cap extract and the "rest" of the root extract were equally efficient in inducing

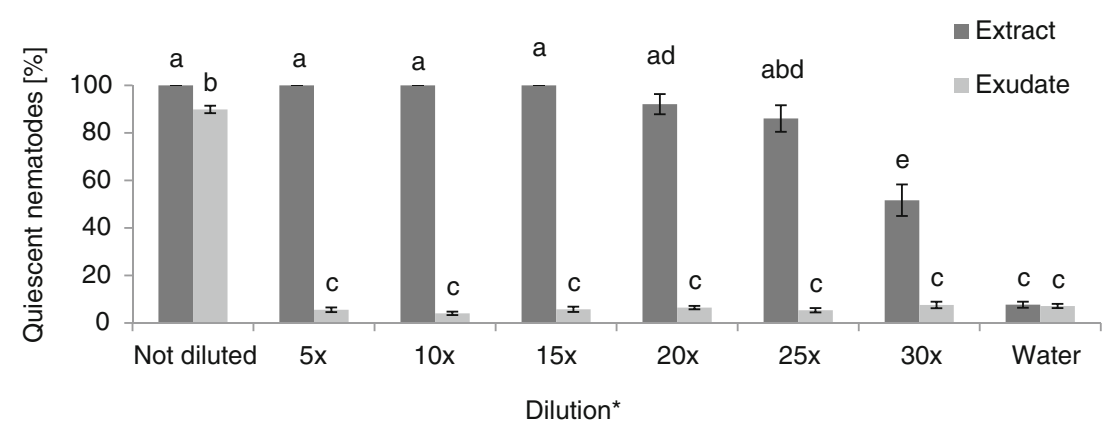

Fig. 1 Differences between pea root cap extract and root cap exudate in inducing quiescence in Heterorhabditis megidis. Overall, extract obtained by grinding the roots was more efficient in inducing quiescence than exudate. Only undiluted exudate induced more quiescence than water, whereas all of the tested extract dilutions induced more quiescence than water. Different small letters indicate statistical differences. Bars indicate standard error. *The values indicate dilutions of the root cap extract and root cap exudate. They do not include the additional $50 \mu$ l of infective juveniles (IJs) suspension that was added 


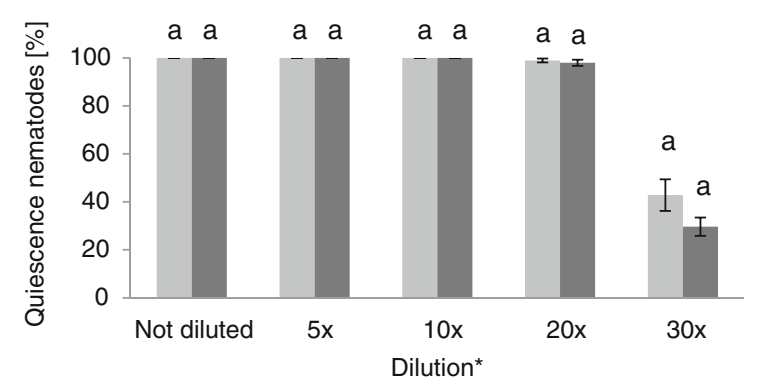

- Tip of the root $(0-10 \mathrm{~mm})$

- Rest of the root $(>10 \mathrm{~mm})$ extract. They do not include the additional $50 \mu 1$ of infective juveniles (IJs) suspension that was added root extracts induced $100 \%$ quiescence until the $1 \mathrm{mg}$ dilution. Small letters indicate statistical differences. Bars indicate standard error. *The

quiescence. The extracts induced $100 \%$ quiescence until a dilution of $10 \times$ for both the root cap and the rest of maize roots. There also was no difference in QF activity between the extracts of the root cap and the extract of the rest of the root for the $\times 20$ and $\times 30$ dilutions (Bonferroni corrected $P$ value $=0.002$; not diluted and $\times 10$ induced $100 \%$ of quiescence; $\times 20$ : $W=274, P=0.68 ; \times 30: W=387, P=0.04$; Fig. 2 ).

Recovery and Infectiousness of $\boldsymbol{H}$. megidis Pea extract: H. megidis exposed to the different pea germinate root cap extract dilutions and water differed in their ability to kill G. mellonella larvae after recovery from quiescence (Weibull model: Chisq $=13.21, P=0.001$; Fig. 3). Overall, $81.3 \%$ of $H$. megidis-exposed larvae were dead at the end of the experiment, and $96.7 \%$ of the dead larvae displayed a red color. Except for the undiluted extract, IJs from all pea germinate extract dilutions were able to kill the host just as well as IJs that were kept in the control condition (water) (not diluted: $Z=2.993, P=0.002 ; \times 10: Z=0.114, P=0.9 ; \times 20: Z=1.469$, $P=0.1 ; \times 30: Z=-0.453, P=0.6$, respectively; Fig. 3 ).

Maize extract: $H$. megidis exposed for $24 \mathrm{hr}$ to the different maize germinate root cap extract dilutions or to just water did not significantly differ in their ability to kill G. mellonella

larvae after recovery from quiescence (Weibull model: Chisq $=6.09, P=0.19)$. Overall, $92 \%$ of the larvae were dead at the end of the experiment, and $89.70 \%$ of the dead larvae displayed a red color. In all maize germinate extract dilutions, the IJs were able to kill G. mellonella larvae just as well as the ones that were kept in the control condition (water) (not diluted: $Z=1.212, P=0.2 ; \times 10: Z=-1.31, P=0.1 ; \times 20: Z=-0.068$, $P=0.9 ; \times 30: Z=-0.564, P=0.5$; Fig. 4). Nevertheless, as for the IJs exposed to pea root extract, the "undiluted" treatment was the one with the lowest number of dead larvae ( $24 \%$ of larvae remained alive at the end of the experiment compared to $0,7.6,6.66$, and $6.66 \%$ for the $\times 10, \times 20, \times 30$ dilutions and water, respectively, Fig. 4).

The results were similar when we used T. molitor larvae. Infective juveniles exposed for $24 \mathrm{hr}$ to the different maize germinate root cap extract dilutions, or to just water did not significantly differ in their ability to kill $T$. molitor larvae after recovery from quiescence (Weibull model: $C h i s q=2.86$, $P=0.58$ ). Overall, $85.3 \%$ of the larvae were dead at the end of the experiment and $90.6 \%$ of the dead larvae displayed a red color. Infective juveniles from all extract concentrations were able to kill $T$. molitor larvae just as well as the ones that were kept in the control condition (water) (not diluted: $Z=0.794$,

Fig. 3 Ability of Heterorhabditis megidis infective juveniles (IJs) to kill Galleria mellonella larvae after incubation in pea root extract ( $24 \mathrm{hr}$ ), as compared to incubation in just water. Nematodes incubated in all extract dilutions except the undiluted extract were as efficient as nematodes kept in water in killing host larvae. Different small letters indicate statistical differences

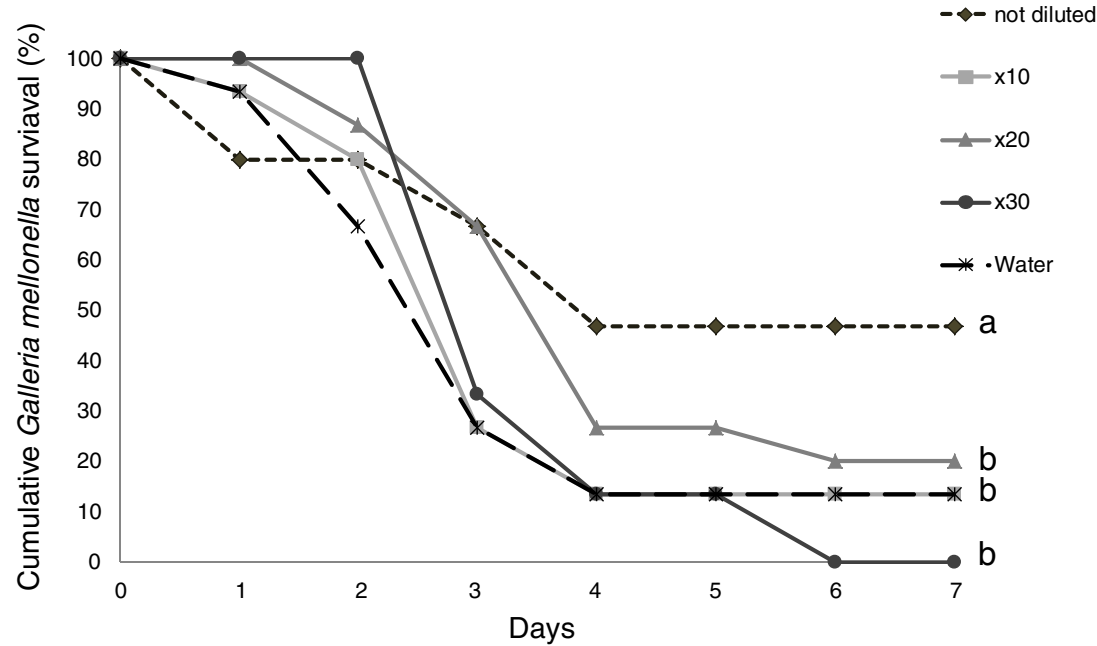


Fig. 4 Ability of Heterorhabditis megidis infective juveniles (IJs) to kill Galleria mellonella larvae after incubation in maize root extract (24 hr), as compared to incubation in just water. Nematodes incubated in all extract dilutions were as efficient as nematodes kept in water in killing host larvae. Different small letters indicate statistical differences

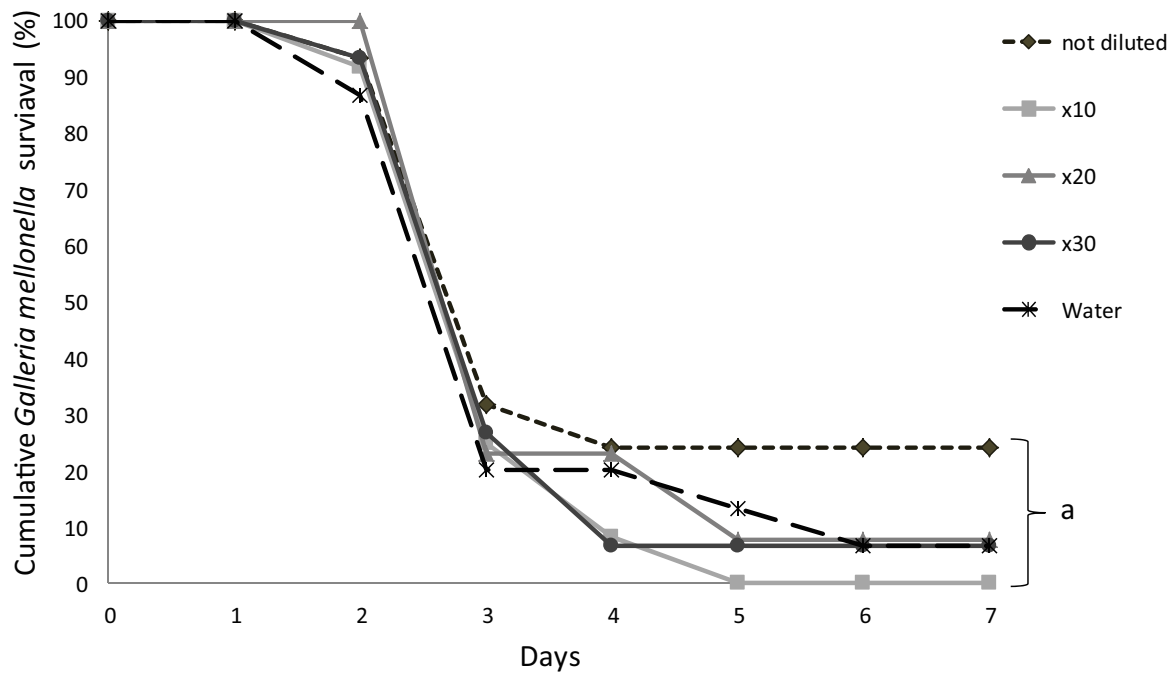

volume (1 ml) of water showed no quiescence, unlike IJs in the same volume of root cap extract $(D F=78, P<0.001)$ (Table 1).

\section{Discussion}

For an optimal EPN formulation, especially in the context of commercialization, a prolonged infectiveness of EPNs is decisive (Grewal 2002). The possibility to induce quiescence in nematodes is a highly promising way to prolong the shelf life of EPNs and to maintain infectiveness for an extended period of time (Hiltpold et al. 2014). Quiescent factor is found in various genera of plants, and triggers a state of quiescence in all types of nematodes. Normally, the QF is collected from the exudate of root caps (Hiltpold et al. 2014; Hubbard et al. 2005). This collection method has several drawbacks, as it is time consuming and a tedious process, and only small quantities can be obtained. In this paper, we present a new and efficient way to collect QF
Fig. 5 Ability of Heterorhabditis megidis infective juveniles (IJs) to kill Tenebrio molitor larvae after incubation in maize root extract ( $24 \mathrm{hr}$ ), as compared to incubation in just water. Nematodes incubated in all extract dilutions were as efficient as nematodes kept in water in killing host larvae. Different small letters indicate statistical differences

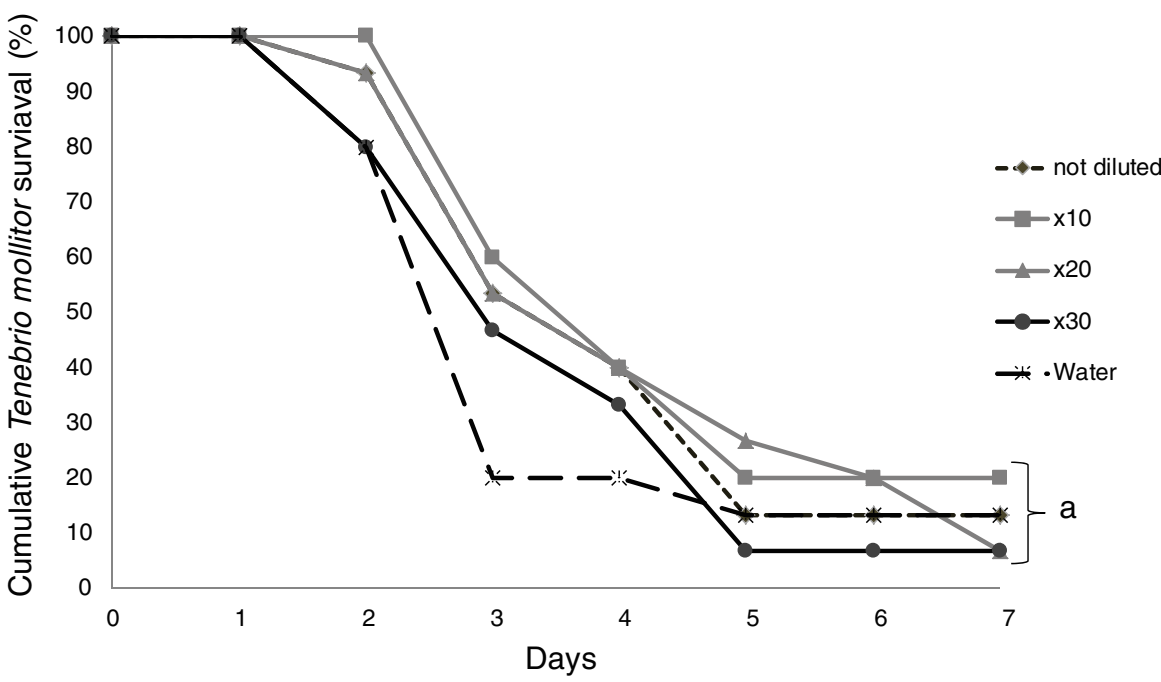


Table 1 Effect of volume, agitation and aeration of extract on Heterorhabditis megidis quiescence

\begin{tabular}{llllll}
\hline Parameter & Volume & & Agitation & & Aeration \\
\hline Treatment & $1 \mathrm{ml}$ extract & $200 \mu \mathrm{l}$ extract & No agitation & Agitation & No aeration \\
H. megidis quiescence & + & - & + & - & Aeration \\
\hline
\end{tabular}

from plants by using extraction of flash-frozen roots in liquid nitrogen. The root extracts that we obtained had high concentrations of QF and in all cases triggered $100 \%$ quiescence at dilutions as low as $0.5 \mathrm{mg}$ for pea root cap and $0.66 \mathrm{mg}$ for maize root cap $(0.5 \mathrm{mg}$, was not tested for maize). In contrast, root cap exudate had only low levels of QF and quickly lost its activity once diluted, which is consistent with the results obtained by Hiltpold et al. (2014).

Previous research on the QF focused on the root cap, and it was not known that the QF also is present in other parts of the root. Here we showed that the QF is constitutively produced in the entire root. The root cap extract and the extract of the "rest" of the root were equally efficient in triggering quiescence in $H$. megidis. This finding implies that considerably more plant material can be used to collect the QF. Whether the other root parts also release the $\mathrm{QF}$ into the rhizosphere remains to be determined, and we cannot exclude the possibility that the quiescence inducing compound(s) present in the rest of the root are different from those in the root cap.

It is important to note that a highly concentrated extract might contain too much QF for EPNs to recover from quiescence. This is important to consider when extracts are used in EPN formulation. We therefore tested the efficiency of the $H$. megidis IJs to recover from the state of quiescence, as well as their infectiousness after quiescence. For this, we used the relatively resistant host species $T$. molitor, as well as the very susceptible model species $G$. mellonella, and found that, after recovery, all the IJs exposed to the different dilutions of pea and maize extract for $24 \mathrm{hr}$ were able to kill both hosts efficiently. The results imply that EPNs, even after exposure to high doses of QF, can be released in crop fields in a state of quiescence and still be able to kill a target pests.

In previous assays, we had noticed that root cap extract did not always trigger quiescence in $H$. megidis IJs. After further investigation, it was found that overexposure to air was possibly responsible for this absence of quiescence. We subsequently showed that quiescence induction by maize root cap extract is efficient only under relatively low oxygen conditions. Indeed, IJs kept in a small volume of root cap extract, representing a thin layer of solution and thus considerable exposure to air, did not turn into a state of quiescence, contrary to IJs that were kept in a larger volume (thick layer). Importantly, IJs kept in a larger volume of root cap extract that was shaken also failed to become quiescent. Aeration of the root cap extract by means of an aquarium pump extract did not have any effect; IJs that were placed in extract that had first been aerated (thick layer) all became quiescent. As aeration did not change the proprieties of the QF in the root cap extract, it is clearly not that the QF is sensitive to exposure to air, but the exposure of EPNs to air interferes with the triggering of quiescence. A possible explanation is that a slightly weakened condition due to lack of oxygen may facilitate the triggering of quiescence. For the identification of the QF, we plan to use liquid chromatography to fractionate the exudate and bioassay guided isolation of the active compound(s) from these fractions. From the current results we learned that for these bioassays it is essential that we maintain the right conditions to ensure that a loss of activity is due only to a loss of active compound(s).

For now, we can only speculate on the ecological role of QFs in nature. It may well be a root defense against phytopathogenic nematodes (PPNs). Indeed, PPNs are considerable more sensitive than EPNs and may not recover from quiescence when exposed to high concentrations of QF (Hiltpold et al. 2014). Even if they recover, "drugging" PPNs may be an effective way for root tips to escape infection (Hiltpold et al. 2014). The eventual identification of QF should shed more light on its function and importance in shaping plantnematode interactions.

In summary, we demonstrated that flash-frozen pea and maize roots extracts are highly effective in inducing quiescence in $H$. megidis, and are far more concentrated in QF than root exudates. At all concentrations, the state of quiescence was reversible after placing the IJs in soil with a high water content, and the IJs were still highly infectious after recovery. However, we did find that there is a limitation of the use of root cap extracts to induce quiescence: induction works only under conditions of slightly reduced oxygen availability. By using the presented method to obtain high concentrations of QF, we hope to be able to identify the key compound(s) that is (are) for quiescence induction.

Acknowledgments We thank the members of the FARCE laboratory for their frequent assistance and relevant discussion. We especially thank Drs. Jinwon Kim and Raquel Campos Herrera for fruitful discussions and their helpful comments on an earlier version of the manuscript. We also thank Andermatt Biocontrol SA (Switzerland), and DSP SA (Switzerland) for providing EPNs and seeds, respectively. This work was supported by an economic stimulus grant from the Swiss National Science Foundation. 


\section{References}

Adams B, Nguyen K (2002) Taxonomy and systematics. In: Gaugler R (ed) Entomopathogenic nematology. CABI, New York, pp 1-34

Ali JG, Alborn HT, Stelinski LL (2010) Subterranean herbivore-induced volatiles released by citrus roots upon feeding by Diaprepes abbreviatus recruit entomopathogenic nematodes. J Chem Ecol 36:361-368. doi:10.1007/s10886-010-9773-7

Ali JG, Alborn HT, Campos-Herrera R, Kaplan F, Duncan LW, Rodriguez-Saona C, Koppenhöfer M, Stellinski LL (2012) Subterranean, herbivore-induced plant volatile increases biological control activity of multiple beneficial nematode species in distinct habitats. PLoS ONE 7:1-8. doi:10.1371/journal.pone.0038146

Bais HP, Weir TL, Perry LG, Gilroy S, Vivanco JM (2006) The role of root exudates in rhizosphere interactions with plants and other organisms. Annu Rev Plant Biol 57:233-266. doi:10.1146/annurev. arplant.57.032905.105159

Barber BYDA, Martin JK, June I (1976) The release of organic substances by cereal roots into soil. New Phytol 76:69-80

Barrett J (1991) Anhydrobiotic nematodes. In: Evans K (ed) Agricultural zoological reviews. Vol. 4. Intercept, Andover, pp 161-176

Chen S, Glazer I (2005) A novel method for long-term storage of the entomopathogenic nematode Steinernema feltiae at room temperature. Biol Control 32:104-110. doi:10.1016/j.biocontrol.2004.08.006

Curtis RHC, Robinson AF, Perry RN (2009) Hatch and host location. In: Perry R, Moen M, Starr J (eds) Root-knot nematodes. CABI Publ, Wallingford, pp 139-162

Den Nijs LJMF, Lock CAM (1992) Differential hatching of the potato cyst nematodes Globodera rostochiensis and G. pallida in root diffusates and water of differing ionic composition. Neth J Plant Pathol 98:117-128. doi:10.1007/BF01996324

Dillman AR, Chaston JM, Adams BJ, Ciche TA, Goodrich-Blair H, Stock SP, Sternberg PW (2012) An entomopathogenic nematode by any other name. PLoS Pathog 8:8-11. doi:10.1371/journal.ppat.1002527

Forst S, Clarke D (2002) Bacteria-nematode symbiosis. In: Gaugler R (ed) Entomopathogenic nematology. CABI Publishing, New York, pp 57-77

Gaur HS, Beane J, Perry RN (2000) The influence of root diffusate, host age and water regimes on hatching of the root-knot nematode, Meloidogyne triticoryzae. Nematology 2:191-199

Georgis R, Koppenhöfer M, Lacey LA, Bélair G, Duncam LW, Grewal PS, Samish M, Tan L, Torr P, van Tol RWHM (2006) Successes and failures in the use of parasitic nematodes for pest control. Biol Control 38:103-123. doi:10.1016/j.biocontrol.2005.11.005

Grewal PS (2002) Formulation and application technology. In: Gaugler R (ed) Entomopathogenic nematology. CABI Publishing, New York, pp 265-287

Grewal PS, Peters A (2005) Formulation and quality control of entomopathogenic nematodes. In: Grewal PS, Ehlers RU, Shapiro-Ilan D (eds) Nematodes as biocontrol agents. CABI Publishing, Wallingford, pp 79-90

Hartmann T (2007) From waste products to ecochemicals: fifty years research of plant secondary metabolism. Phytochemistry 68:28312846. doi:10.1016/j.phytochem.09.017

Hiltpold I, Turlings TCJ (2012) Manipulation of chemically mediated interactions in agricultural soils to enhance the control of crop pests and to improve crop yield. J Chem Ecol 38:641-650. doi:10.1007/ s10886-012-0131-9

Hiltpold I, Erb M, Robert CAM, Turlings TCJ (2011) Systemic root signalling in a belowground, volatile-mediated tritrophic interaction. Plant Cell Environ 34:1267-1275. doi:10.1111/j.1365-3040.2011.02327.x

Hiltpold I, Hibbard BE, French BW, Turlings TCJ (2012) Capsules containing entomopathogenic nematodes as a Trojan horse approach to control the western corn rootworm. Plant Soil 358:11-25. doi:10. 1007/s11104-012-1253-0
Hiltpold I, Jaffuel G, Turlings TCJ (2014) The dual effects of root-cap exudates on nematodes: from quiescence in plant-parasitic nematodes to frenzy in entomopathogenic nematodes. J Exp Bot. doi: $10.1093 / \mathrm{jxb} / \mathrm{eru} 345$

Hubbard JE, Flores-Lara Y, Schmitt M, McCLure MA, Stock SP, Hawes MC (2005) Increased penetration of host roots by nematodes after recovery from quiescence induced by root cap exudate. Nematology 7:321-331

Kaya HK, Gaugler R (1993) Entomopathogenic nematodes. Annu Rev Entomol 38:181-206. doi:10.1146/annurev.en.38.010193.001145

Khokon MAR, Okuma E, Rahman T, Wesemael WML, Murata Y, Moens M (2009) Quantitative analysis of the effects of diffusates from plant roots on the hatching of Meloidogyne chitwoodi from young and senescing host plants. Biosci Biotechnol Biochem 73:2345-2347. doi:10.1271/bbb.90392

Köhler HR, Triebskorn R (2013) Wildlife ecotoxicology of pesticides: can we track effects to the population level and beyond? Science 341:759-765. doi:10.1126/science.1237591

Kumar R, Pandey S, Pandey A (2006) Plant roots and carbon sequestration. Curr Sci 91:885-890

Lello ER, Patel MN, Matthews G, Wright DJ (1996) Application technology for entomopathogenic nematodes against foliar pests. Crop Prot 15:567-574. doi:10.1016/0261-2194(96)00026-9

Lewis EE, Campbell J, Griffin C, Kaya H, Peters A (2006) Behavioral ecology of entomopathogenic nematodes. Biol Control 38:66-79. doi:10.1016/j.biocontrol.2005.11.007

Prot J (1980) Migration of plant-parasitic nematodes towards plant roots. Rev Nemtology 3(2): 305-318

Rasmann S, Köllner TG, Degenhardt J, Hiltpold I, Toepfer S, Kuhlmann U, Gershenton J, Turlings TCJ (2005) Recruitment of entomopathogenic nematodes by insect-damaged maize roots. Nature 434:732737. doi:10.1038/nature 03451

Rasmann S, Hiltpold I, Ali J (2012) The role of root-produced volatile secondary metabolites in mediating soil interactions. In: Montanaro G, Cichio B (eds) Advances in selected plant physiology aspects. InTech Open Access Publisher, Croatia, pp 269-290

Reynolds AM, Dutta TK, Curtis RHC, Powers SJ, Gaur HS, Kerry BR (2011) Chemotaxis can take plant-parasitic nematodes to the source of a chemo-attractant via the shortest possible routes. J R Soc Interface 8:568-577. doi:10.1098/rsif.2010.0417

Rolfe RN, Barrett J, Perry ARN (2000) Analysis of chemosensory responses of second stage juveniles of Globodera rostochiensis using electrophysiological techniques. Nematology 2:523-533

Shapiro-Ilan DI, Gouge DH, Piggott SJ, Fife JP (2006) Application technology and environmental considerations for use of entomopathogenic nematodes in biological control. Biol Control 38:124-133. doi:10.1016/j.biocontrol.2005.09.005

Turlings TCJ, Hiltpold I, Rasmann S (2012) The importance of rootproduced volatiles as foraging cues for entomopathogenic nematodes. Plant Soil 358:51-60. doi:10.1007/s11104-012-1295-3

Van Dam NM (2009) Belowground herbivory and plant defenses. Annu Rev Ecol Evol Syst 40:373-391. doi:10.1146/annurev.ecolsys. 110308.120314

Van Tol RWHM, Van der Sommen ATC, Boff MIC, Van Bezooijen J, Sabelis MW, Smits PH (2001) Plants protect their roots by alerting the ennemies of grubs. Ecol Lett 4:292-294. doi:10.1046/j.14610248.2001.00227.x

Vining LC (1990) Functions of secondary metabolites. Annu Rev Microbiol 44:395-427. doi:10.1146/annurev.mi.44.100190.002143

White GF (1927) A method for obtaining infective nematode larvae from cultures. Science 66:302-303

Zhao X, Schmitt M, Hawes MC (2000) Species-dependent effects of border cell and root tip exudates on nematode behavior. Phytopathology 90:1239-1245. doi:10.1094/PHYTO.2000.90.11. 1239 